\title{
Comparison of Rates and Outcomes of Readmission to Index vs Non-index Hospitals After Intravenous Thrombolysis in Acute Stroke Patients
}

Kristina Shkirkova ${ }^{1}$, Michelle Connor ${ }^{2}$, Drew M. Hodis ${ }^{3}$, Krista Lamorie-Foote $^{2}$, Arati Patel ${ }^{4}$, Qinghai $\mathrm{Liu}^{2}$, Li Ding ${ }^{5}$, Arun Amar ${ }^{2}$, Nerses Sanossian ${ }^{6}$, Frank Attenello ${ }^{1}$, William Mack ${ }^{2}$

1. Neurological Surgery, University of Southern California, Keck School of Medicine, Los Angeles, USA 2. Neurological Surgery, University of Southern California, Los Angeles, USA 3. Zilkha Neurogenetic Institute, University of Southern California, Los Angeles, USA 4. Neurological Surgery, University of California, San Francisco, San Francisco, USA 5. Preventive Medicine, University of Southern California, Los Angeles, USA 6. Neurology, University of Southern California, Los Angeles, USA

Corresponding author: Kristina Shkirkova, kristina.shkirkova@usc.edu

\begin{abstract}
National and regional systems of stroke care are designed to provide patients with widespread access to hospitals with thrombolytic capabilities. However, such triaging systems may contribute to fragmentation of care. This study aims to compare rates of readmission and outcomes between index and non-index hospitals for stroke patients following intravenous thrombolytic therapy (IVT). This study utilized a nationally representative sample of stroke patients with IVT from the Nationwide Readmissions Database from 2010 to 2014. Descriptive and regression analyses were performed for patient and hospital level factors that influenced 90-day readmissions and regression models were used to identify differences in mortality, complications, and repeat readmissions between patients readmitted to index (facility where IVT was administered) and non-index hospitals. In the study, 49415 stroke patients were treated with IVT, of whom $21.7 \%$ were readmitted within 90 days. Among readmissions, $79.4 \%$ of patients were readmitted to index hospitals and $20.6 \%$ to non-index hospitals. On multivariate logistic regression analysis, index hospital readmission was independently associated with lower frequency of second readmissions (non-index OR $1.09,95 \%$ CI 1.07-1.11, $\mathrm{p}<0.0001$ ) but not with increased mortality or major complications ( $\mathrm{p}=\mathrm{ns})$. Approximately one-fifth of stroke patients treated with thrombolysis were readmitted within 90 days, onefifth of whom were readmitted to non-index hospitals. Although readmission to index hospital was associated with lower frequency of subsequent readmissions, readmission to non-index hospital was not associated with increased mortality or major complications. This difference may be due to standardized algorithms, mature systems of care, and demanding metrics required of stroke centers.
\end{abstract}

Received 02/24/2020 Review began 02/26/2020 Review ended 06/25/2020 Published 07/01/2020

() Copyright 2020 Shkirkova et al. This is an open access article distributed under the terms of the Creative Commons Attribution License CC-BY 4.0., which permits unrestricted use, distribution, and reproduction in any medium, provided the original author and source are credited.
Categories: Neurology, Quality Improvement, Public Health

Keywords: stroke, thrombolysis, readmission, stroke systems of care

\section{Introduction}

An estimated 795,000 people suffer from stroke in the United States each year [1]. Eighty-seven percent of these strokes are ischemic. Management of ischemic stroke is time-sensitive as clinical symptoms often progress rapidly to permanent neurological deficits. Pre-hospital neurological deterioration occurs in $9 \%$ of ischemic stroke patients [2]. Roughly 7\% of patients deteriorate during the early Emergency Department (ED) phase [2], and approximately 20\% experience neurological worsening within the first 24 to 48 hours at the hospital [3]. Vessel recanalization and restoration of perfusion to viable brain tissue is the most effective means of limiting ischemic stroke progression. Originally approved in 1996 for use in the United States after the positive National Institute of Neurological Disorders and Stroke (NINDS) trial [4], thrombolysis with intravenous tissue plasminogen activator (IV-tPA) is considered to be the standard of care for eligible patients suffering from acute ischemic stroke [4]. The recommended window for IV-tPA treatment is within 3 , or in some cases 4.5 , hours after the onset of stoke [5, 6]. It is, therefore, critical for ischemic stroke patients to be brought to a capable stroke center in rapid fashion following onset of symptoms.

National and regional systems of stroke care aim to provide patients with widespread access to hospitals with thrombolytic capabilities, as early intervention with IV-tPA is critical for improved outcomes. Emergency Medical Services (EMS) protocols dictate routing of suspected stroke patients to certified stroke hospitals such as Primary Stroke Centers (PSCs) to expedite in-hospital time-to-treatment processes. However, ischemic stroke patients treated with IV-tPA have risks of disease- and treatment- related complications, as well as comorbidities, that typically require specialized in-hospital and follow-up management $[1,7,8]$. Stroke systems and routing parameters often result in patients initially being treated in hospitals that are further from their homes. This may negatively impact continuity of care and management of complications in cases of readmission (potentially to a different hospital) [9]. 
In a number of medical conditions, readmission to a different hospital is associated with less favorable outcomes due to care fragmentation $[10,11]$. Our study leverages the Nationwide Readmission Database to determine rates of readmission to index (site of first admission) and non-index (site other than first admission) hospitals for stroke patients following thrombolysis therapy and to compare readmission outcomes between the index and non-index readmission types.

\section{Materials And Methods}

\section{Data Source}

The Healthcare Cost and Utilization Project Nationwide Readmissions Database (NRD) is a nationally representative database with information about patients with readmissions following initial hospitalization. The NRD database collects data from 28 states, representing about $58 \%$ of all hospitalizations and readmissions in the US. The NRD provides a unique identifier link for each patient, within each calendar year, within each state, that is used to determine patients' hospitalizations within that year. The index hospital was defined as the hospital at which the patient was initially admitted and treated with IVT, while a non-index hospital was defined as any other hospital. The NRD is a de-identified, publicly available database. No institutional review board or ethics approval was required for this study. This study is a retrospective analysis of the NRD database from 2010-2014.

\section{Inclusion and Exclusion Criteria}

International Classification of Disease, Ninth Edition, Clinical Modifications (ICD-9-CM) diagnosis and procedure codes were used to identify patients meeting inclusion criteria. Adult patients ( $\geqslant 18$ years old) with a diagnosis of ischemic stroke (433.01, 433.11, 433.21, 433.31, 433.81, 433.91, 434.01, 434.11, 434.91, 436) managed with IVT (99.10, V45.88) were selected from the NRD and included in this study. Only non-elective admissions were considered in this study. As the NRD records patient data for a single calendar year, only patients with sufficient follow-up time of 90 days (discharged between January and September) were included in this study. Only the first readmission within 90-days was included in this analysis. However, rates of repeat readmission were measured.

Patients with a diagnosis of head trauma (80x.xx, 85x.xx), subarachnoid hemorrhage (430), extra-axial hematoma (432.0, 432.1, 432.9), arteriovenous malformation (747.81), cerebral arteritis (437.4), Moyamoya disease (437.5), venous sinus thrombosis (437.6), brain tumor (191.x, 192.0, 192.1, 194.3, 198.3, 198.4, 199.0, 200.5, 225.x, 227.3, 237.0, 237.5, 237.6), or intracranial abscess (324.0) were excluded. Additionally, patients who underwent microsurgical clipping of a cerebral aneurysm $(39.51,39.52)$, repair of an arteriovenous malformation (39.53), cranioplasty (02.03-02.07), stereotactic radiosurgery (92.3x), carotid artery stent placement (00.63), or carotid endarterectomy (38.12) were excluded. Patients who died during the index hospitalization, or who were missing "died" or "length of stay" information were excluded. Patients identified according to the above inclusion and exclusion criteria were included as study participants.

\section{Association Variables}

Patient and hospital level characteristics were included in the analysis. Patient demographics included age (categorized as 18-44, 45-59, 60-74, >75), gender (male or female), insurance type (Medicare, Medicaid, private, self-pay, no charge, or other), median household income (by quartile), residency status (resident/nonresident of state where procedure was performed), discharge quarter (January-March, AprilJune, July-September), and discharge disposition (routine vs other). Other patient characteristics included comorbidity score, major complications, neurological complications, and second readmission. Comorbidity score was determined by the Elixhauser variables and re-categorized into 3 groups $(0-1,2$, or $\geqslant 3)$. Major complications included pneumonia (481-482, 482.1-482.3, 482.30-482.32, 482.39-482.41, 482.49, 482.80$482.84,482.89,482.90,483.0,483.1,483.8,485-487.0,997.3,507.0)$, pulmonary embolism (415.1-415.9), renal failure $(584,584.5-584.9)$, cerebrovascular accident $(433.01,433.11,433.21,433.31,433.81,433.91)$, myocardial infarction (410.00-410.90, 410.01, 410.11-410.91), cardiac arrest (427.5), sepsis (995.91), and septic shock (995.92). Neurological complications included intracerebral hemorrhage (431, 998.11-998.12), seizures (345.xx), and neurological complications after procedure (997.01-997.09). All patient-refined diagnosis related groups (APR-DRG) severity of illness and risk of mortality scores (minor, moderate, major, extreme) provided by NRD were also included. Hospital characteristics included bed size (small, medium, or large), academic vs non-academic status, ownership status (government, nonfederal; private, nonprofit; private, invest-own), and urban vs rural location.

\section{Outcome Variables}

Rates of readmission to index or non-index hospitals within 90 days of initial hospitalization were quantified. Reasons for readmission, based on ICD-9-CM codes, were identified. Patient and hospital factors associated with readmission were evaluated. Outcomes following readmission to index or non-index hospitals, including mortality, major complications, neurological complications, and second readmission were assessed. Factors associated with mortality, major complications, neurological complications, and second readmission were identified. 


\section{Statistical Analysis}

Descriptive analyses were performed to characterize patient and hospital factors associated with 90-day readmission to index or non-index hospitals. Multivariable logistic regression models were used for mortality, major complications, neurological complications, and frequency of second readmissions. Patient and hospital level demographic variables from the initial hospitalization (listed above) were included in the models. A sensitivity analysis was performed to adjust for readmission hospital characteristics. Data was reported using odds ratios (OR) and 95\% confidence intervals (CI). Statistical significance was defined as $\mathrm{p}<0.05$. Statistical analysis was conducted using SAS 9.4 (SAS Inc., Cary, North Carolina, USA).

\section{Results}

\section{Study Participants and Descriptive Data}

A total of 49415 patients with ischemic stroke were treated with IV-tPA during the study period. Among them, 10718 (21.7\%) patients were readmitted within 90 days, which constituted the primary population of this study. Of the 10718 readmitted patients, 8514 (79.4\%) were readmitted to the index hospital and 2204 (20.6\%) were readmitted to a non-index hospital. Age distribution of the study population was: 18-44 (4.8\%), 45-59 (17.8\%), 60-74 (32.5\%), $\geqslant 75$ (44.8\%). Percentage of female patients was $49.9 \%$. (Table 1 ).

\begin{tabular}{|c|c|c|c|c|}
\hline & $\begin{array}{l}\text { Total } \\
(n=10718)\end{array}$ & $\begin{array}{l}\text { Index Hospital } \\
(n=8514)\end{array}$ & $\begin{array}{l}\text { Non-index Hospital } \\
(n=2204)\end{array}$ & p-value \\
\hline \multicolumn{5}{|l|}{ Age, n (\%) } \\
\hline $18-44$ & $517(4.8)$ & $387(4.6)$ & $130(5.9)$ & \multirow{4}{*}{0.0007} \\
\hline $45-59$ & $1910(17.8)$ & 1489 (17.5) & $421(19.1)$ & \\
\hline $60-74$ & 3486 (32.5) & 2747 (32.3) & $739(33.5)$ & \\
\hline$\geq 75$ & $4805(44.8)$ & $3891(45.7)$ & $914(41.5)$ & \\
\hline Female, n (\%) & $5349(49.9)$ & $4263(50.1)$ & 1086 (49.3) & 0.51 \\
\hline \multicolumn{5}{|c|}{ Primary Insurance, n (\%) } \\
\hline Medicare & $7357(68.8)$ & 5899 (69.3) & $1476(67.0)$ & \multirow{5}{*}{0.0071} \\
\hline Medicaid & $977(9.1)$ & 733 (8.6) & $244(11.1)$ & \\
\hline Private insurance & $1711(16.0)$ & $1365(16.0)$ & $346(15.7)$ & \\
\hline Self-pay & 358 (3.3) & 278 (3.3) & $80(3.6)$ & \\
\hline No charge & $43(0.4)$ & $38(0.5)$ & $\mathrm{DS}^{*}(0.2)$ & \\
\hline Other & $234(2.2)$ & $186(2.2)$ & $48(2.2)$ & \\
\hline Missing & $20(0.2)$ & $15(0.2)$ & $\mathrm{DS}^{\star}(0.2)$ & \\
\hline \multicolumn{5}{|c|}{ Comorbidity Score, n (\%) } \\
\hline 0 & 209 (1.9) & $162(1.9)$ & $47(2.1)$ & \multirow{4}{*}{0.22} \\
\hline 1 & 995 (9.3) & $782(9.2)$ & $213(9.7)$ & \\
\hline 2 & $1868(17.4)$ & $1515(17.8)$ & $353(16.0)$ & \\
\hline 3 & 7646 (71.3) & 6055 (71.1) & 1591 (72.2) & \\
\hline \multicolumn{5}{|c|}{ Median Household Income*, n (\%) } \\
\hline $0-25$ percentile & $2961(27.6)$ & $2304(27.1)$ & $657(29.8)$ & \multirow{5}{*}{0.025} \\
\hline 26-50 percentile & $2568(24.0)$ & $2027(23.8)$ & $541(24.6)$ & \\
\hline 51-75 percentile & $2585(24.1)$ & 2072 (24.3) & $513(23.3)$ & \\
\hline 76-100 percentile & 2445 (22.8) & 1979 (23.2) & $466(21.1)$ & \\
\hline Missing & $159(1.5)$ & $132(1.6)$ & 27 (1.2) & \\
\hline
\end{tabular}




\section{Cureus}

Minor
Moderate
Major
Extreme
All Patient Refined DRG: Severity of Illness Subclass, $n(\%)$

$\begin{array}{lll}2324(21.7) & 1827(21.5) & 497(22.6) \\ 4085(38.1) & 3293(38.7) & 792(35.9) \\ 2485(23.2) & 1986(23.3) & 499(22.6) \\ 1824(17.0) & 1408(16.5) & 416(18.9)\end{array}$

Minor

$465(4.3) \quad 365(4.3)$

$100(4.5)$

Moderate

4114 (38.4)

$3299(38.8)$

$815(37.0)$

$4245(39.6) \quad 3382(39.7)$

863 (39.2)

Major

1894 (17.7)

$1468(17.2)$

426 (19.3)

Control/Ownership of Hospital, $\mathrm{n}(\%)$

Government, non-federal

$120(22.9)$

87 (22.5)

33 (24.2)

Private, non-profit

7648 (71.4)

$6126(72.0)$

$1522(69.1)$

0.0123

Private, invest-own

1509 (14.1)

$1188(14.0)$

$321(14.6)$

Teaching Status of Urban Hospitals, $n$ (\%)

Teaching

$6608(61.7)$

$5198(61.1)$

$1410(64.0)$

Non-teaching

4110 (38.3)

$3316(39.0)$

0.0119

Hospital Bed Size, n (\%)

Small

Medium

$629(5.9) \quad 474(5.6)$

$2327(21.7) \quad 1856(21.8)$

0.0331

Large

$7762(72.4) \quad 6184(72.6)$

$471(21.4)$

0.0155

0.11

Hospital Urban-Rural Designation, n (\%)

Large metropolitan areas with at least 1 million residents, $\mathrm{n}$ (\%)

$6583(61.4) \quad 5134(60.3)$

$1449(65.7)$

$4135(38.6) \quad 3380(39.7)$

$<0.0001$

Other

755 (34.3)

Resident of state where procedure was performed, $\mathrm{n}(\%)$

Nonresident

334 (3.1) $\quad 276(3.2)$

58 (2.6)

Resident

10384 (96.9)

$8238(96.8)$

Discharged to Another Facility, n (\%)

Yes

No

$7475(69.7) \quad 5900$ (69.3)

$2146(97.4)$

Major Complication, n (\%)

Yes

$3174(29.6) \quad 2514(29.5)$

$660(30.0)$

$7544(70.4) \quad 6000(70.5)$

0.70

No

Neurological Complication, n (\%)

Yes

$1300(12.1) \quad 993(11.7)$

307 (13.9)

9418 (87.9) $7521(88.3)$

1897 (86.1)

0.0037

No

Repeat Readmission, n (\%)

Yes

$2572(24.0) \quad 1882(22.1)$

690 (31.3)

No

$8146(76.0) \quad 6632(77.9)$

1514 (68.7)

$<0.0001$

Discharge Quarter, n (\%) 


\section{Cureus}

$\begin{array}{lllll}\text { Jan-March } & 3501(32.7) & 2773(32.6) & 728(33.0) & \\ \text { April-June } & 3562(33.2) & 2843(33.4) & 719(32.6) & 0.79 \\ \text { July-Sep } & 3655(34.1) & 2898(34.0) & 757(34.4) & \end{array}$

\section{TABLE 1: Patient and Hospital Characteristics at Index and Non-index 90-Day Readmissions}

* for patient's ZIP code, based on current year

DS ${ }^{\star}$ - data with less than 10 patients is suppressed

The most common reasons for readmission were cerebral artery occlusion (8.73\%), septicemia (5.83\%), and carotid artery occlusion (5.22\%) (Table 2).

\begin{tabular}{|c|c|c|}
\hline ICD & Diagnosis & $n(\%)$ \\
\hline 43491 & Cerebral Artery Occlusion w/ Infarct & $935(8.7)$ \\
\hline 0389 & Septicemia & $624(5.8)$ \\
\hline 43310 & Carotid Artery Occlusion w/out Infarct & $559(5.2)$ \\
\hline 4359 & Transient Cerebral Ischemia & $311(2.9)$ \\
\hline 5990 & Urinary Tract Infection & $308(2.9)$ \\
\hline 42731 & Atrial Fibrillation & $267(2.5)$ \\
\hline 5070 & Food/Nomit Pneumonitis & $257(2.4)$ \\
\hline 43411 & Cerebral Embolism w/ Infarction & $205(1.9)$ \\
\hline V5789 & Rehabilitation Procedure & $204(1.9)$ \\
\hline 36 & neumonia & $195(1.8)$ \\
\hline
\end{tabular}

TABLE 2: Most frequent reasons for 90-day readmission

\section{Readmission to Index or Non-Index Hospitals}

In the multivariable logistic regression model of index vs non-index readmission, older age (60-74 years old: OR $0.78,95 \%$ CI $0.63-0.97, \mathrm{p}=0.03$; $\geqslant 75$ years old: OR $0.66,95 \%$ CI $0.53-0.83, \mathrm{p}=0.0003$ ) and higher median household income (51-75 percentile: OR $0.85,95 \%$ CI $0.74-0.98, \mathrm{p}=0.02 ; 76-100$ percentile: OR $0.76,95 \% \mathrm{CI}$ $0.66-0.88, \mathrm{p}=0.0003)$ were associated with readmission to index hospital. Non-routine discharge (OR 1.19, 95\% CI 1.06-1.33, $\mathrm{p}=0.002$ ) and neurological complication (OR 1.19, 95\% CI 1.03-1.36, $\mathrm{p}=0.01$ ) were associated with readmission to a non-index hospital. Among hospital level factors, hospital location in a rural area (OR 0.77, 95\% CI 0.69-0.86, p<0.0001) was associated with readmission to index hospital, while small hospital bed size (OR 1.34, 95\% CI 1.11-1.62, $\mathrm{p}=0.003$ ) was associated with readmission to a non-index hospital.

\section{Outcomes following Index vs Non-Index Hospital Readmission}

Readmission to non-index hospital was associated with increased frequency of repeat readmissions (OR $1.09,95 \%$ CI 1.06-1.11, $\mathrm{p}<0.0001)$. Readmission to a non-index hospital was not associated with mortality (OR 1.00, 95\% CI 0.99-1.01, $\mathrm{p}=0.44$ ), major complications (OR 1.00, 95\% CI 0.98-1.02, $\mathrm{p}=0.84$ ), or neurological complications (OR 1.01, 95\% CI 0.99-1.02, p=0.32) (Table 3). 


\section{Cureus}

\begin{tabular}{|c|c|c|c|c|}
\hline Outcome & Index Hospital & Non-index Hospital & OR $(95 \% \mathrm{Cl})$ & p-value \\
\hline Mortality & Reference & 1.00 & 0.99-1.01 & 0.44 \\
\hline Major Complication & Reference & 1.00 & 0.98-1.02 & 0.84 \\
\hline Neurological Complication & Reference & 1.01 & 0.99-1.02 & 0.32 \\
\hline Repeat Readmission & Reference & 1.09 & 1.07-1.11 & $<0.0001$ \\
\hline
\end{tabular}

\section{TABLE 3: Summary Readmission to Index vs Non-index Hospitals Associations}

The multivariate model is additionally adjusted for age, sex, insurance status, discharge quarter, hospital volume, risk of mortality, disease severity, major complications during hospital stay, length of stay, and hospital ownership

\section{Outcomes following Readmission in Overall Index and Non-Index Group}

Patient and hospital factors associated with mortality, major complications, neurological complications, and repeat readmission were assessed. Patient factors associated with mortality included increased age, higher risk of mortality defined as an APR-DRG classification of major or extreme, neurological complication, and discharge to another facility (Table 4 ).

\begin{tabular}{|c|c|c|c|}
\hline & OR & $95 \% \mathrm{Cl}$ & p-value \\
\hline \multicolumn{4}{|l|}{ Hospital } \\
\hline Index hospital & Ref & & \\
\hline Non-index hospital & 1.00 & $0.99-1.01$ & 0.44 \\
\hline \multicolumn{4}{|l|}{ Age } \\
\hline $18-44$ & Ref & & \\
\hline 45-59 & 1.01 & $1.00-1.02$ & 0.02 \\
\hline $60-74$ & 1.02 & $1.01-1.03$ & $<0.0001$ \\
\hline$\geq 75$ & 1.05 & $1.03-1.06$ & $<0.0001$ \\
\hline \multicolumn{4}{|c|}{ All Patient Refined DRG: Risk of Mortality } \\
\hline Minor & Ref & & \\
\hline Moderate & 1.00 & $0.99-1.01$ & 0.81 \\
\hline Major & 1.04 & $1.03-1.05$ & $<0.0001$ \\
\hline Extreme & 1.17 & $1.15-1.20$ & $<0.0001$ \\
\hline \multicolumn{4}{|c|}{ Discharged to Another Facility } \\
\hline Yes & 1.02 & $1.01-1.03$ & $<0.0001$ \\
\hline No & Ref & & \\
\hline \multicolumn{4}{|c|}{ Neurological Complication } \\
\hline Yes & 0.98 & $0.96-0.99$ & 0.001 \\
\hline No & Ref & & \\
\hline
\end{tabular}

\section{TABLE 4: Summary of Associations with Mortality}

The multivariate model is additionally adjusted for age, sex, insurance status, discharge quarter, hospital volume, risk of mortality, disease severity, major complications during hospital stay, length of stay, and hospital ownership 


\section{Cureus}

Increased age, male gender, greater illness severity (APR-DRG of moderate, major, extreme), and presence of a major complication at initial hospitalization were associated with major complications. Presence of a neurological complication at initial hospitalization and shorter length of stay were associated with a lower rate of major complications (Table 5).

\begin{tabular}{|c|c|c|c|}
\hline & OR & $95 \% \mathrm{Cl}$ & p-value \\
\hline \multicolumn{4}{|l|}{ Hospital } \\
\hline Index hospital & \multicolumn{3}{|l|}{ Ref } \\
\hline Non-index hospital & 1.00 & 0.98-1.02 & 0.84 \\
\hline \multicolumn{4}{|l|}{ Age } \\
\hline $18-44$ & Ref & & \\
\hline $45-59$ & 1.01 & 0.98-1.05 & 0.40 \\
\hline $60-74$ & 1.07 & $1.04-1.10$ & $<0.0001$ \\
\hline$\geq 75$ & 1.13 & $1.10-1.17$ & $<0.0001$ \\
\hline \multicolumn{4}{|l|}{ Gender } \\
\hline Male & 1.04 & $1.03-1.06$ & $<0.0001$ \\
\hline Female & Ref & & \\
\hline \multicolumn{4}{|c|}{ All Patient Refined DRG: Disease Severity } \\
\hline Minor & Ref & & \\
\hline Moderate & 1.07 & 1.05-1.09 & $<0.0001$ \\
\hline Major & 1.30 & $1.27-1.33$ & $<0.0001$ \\
\hline Extreme & 1.70 & $1.65-1.76$ & $<0.0001$ \\
\hline \multicolumn{4}{|l|}{ Major Complication } \\
\hline Yes & 1.08 & 1.06-1.10 & $<0.0001$ \\
\hline No & Ref & & \\
\hline \multicolumn{4}{|c|}{ Neurological Complication } \\
\hline Yes & 0.96 & $0.94-0.98$ & 0.001 \\
\hline No & Ref & & \\
\hline \multicolumn{4}{|l|}{ Length of Stay } \\
\hline Per 1 day increase & 1.00 & $1.00-1.00$ & 0.0003 \\
\hline
\end{tabular}

TABLE 5: Summary of Associations with Major Complication

The multivariate model is additionally adjusted for age, sex, insurance status, discharge quarter, hospital volume, risk of mortality, disease severity, major complications during hospital stay, length of stay, and hospital ownership

Illness severity (APR-DRG of moderate, major, extreme), neurological complication at initial hospitalization, and discharge to another facility were associated with higher rate of neurological complication at readmission (Table o). 


\section{Cureus}

\begin{tabular}{|c|c|c|c|}
\hline & OR & $95 \% \mathrm{Cl}$ & $p$-value \\
\hline \multicolumn{4}{|l|}{ Hospital } \\
\hline Index hospital & Ref & & \\
\hline Non-index hospital & 1.01 & $0.99-1.02$ & 0.32 \\
\hline \multicolumn{4}{|l|}{ Age } \\
\hline $18-44$ & Ref & & \\
\hline $45-59$ & 1.00 & $0.97-1.03$ & 0.96 \\
\hline $60-74$ & 0.98 & $0.95-1.01$ & 0.18 \\
\hline$\geq 75$ & 0.95 & $0.93-0.98$ & 0.0002 \\
\hline \multicolumn{4}{|c|}{ All Patient Refined DRG: Disease Severity } \\
\hline Minor & Ref & & \\
\hline Moderate & 1.04 & 1.02-1.05 & $<0.0001$ \\
\hline Major & 1.06 & 1.04-1.07 & $<0.0001$ \\
\hline Extreme & 1.08 & $1.06-1.11$ & $<0.0001$ \\
\hline \multicolumn{4}{|l|}{ Major Complication } \\
\hline Yes & 0.98 & $0.97-0.99$ & 0.002 \\
\hline No & Ref & & \\
\hline \multicolumn{4}{|c|}{ Neurological Complication } \\
\hline Yes & 1.24 & $1.21-1.28$ & $<0.0001$ \\
\hline No & Ref & & \\
\hline \multicolumn{4}{|c|}{ Discharged to Another Facility } \\
\hline Yes & 1.02 & $1.00-1.03$ & 0.011 \\
\hline No & Ref & & \\
\hline
\end{tabular}

\section{TABLE 6: Summary of Associations with Neurological Complication}

The multivariate model is additionally adjusted for age, sex, insurance status, discharge quarter, hospital volume, risk of mortality, disease severity, major complications during hospital stay, length of stay, and hospital ownership

Repeat readmission was associated with comorbidity score $>3$, APR-DRG disease severity score of major, and longer length of stay. Older age, private insurance, household income in the 76-100 percentile, being a nonresident of the state where the procedure was performed, and rural hospital location were associated with lower frequency of repeat readmission (Table 7).

\begin{tabular}{|c|c|c|c|}
\hline & OR & $95 \% \mathrm{Cl}$ & $p$-value \\
\hline \multicolumn{4}{|l|}{ Hospital } \\
\hline Index hospital & \multicolumn{3}{|l|}{ Ref } \\
\hline Non-index hospital & 1.09 & 1.07-1.11 & $<0.0001$ \\
\hline \multicolumn{4}{|l|}{ Age } \\
\hline $18-44$ & Ref & & \\
\hline $45-59$ & 0.99 & $0.95-1.03$ & 0.60 \\
\hline $60-74$ & 0.96 & $0.91-1.00$ & 0.04 \\
\hline
\end{tabular}




\section{Cureus}

$\geq 75$

Primary Insurance

Medicaid

Medicare

Private insurance

Self-pay

Other

Comorbidity Score

Median Household Income

26-50 percentile

51-75 percentile

All Patient Refined DRG: Disease Severity

Minor

Moderate

Major

Extreme

Hospital urban-rural designation

Large metropolitan area with at least 1 million residents

Other

Resident of state where procedure was performed

Nonresident

Resident

Length of Stay

Per 1 day increase

\section{Ref}

\section{TABLE 7: Summary of Associations with Repeat Readmission}

The multivariate model is additionally adjusted for age, sex, insurance status, discharge quarter, hospital volume, risk of mortality, disease severity, major complications during hospital stay, length of stay, and hospital ownership

Sensitivity analysis adjusting for characteristics of readmission hospitals was performed, and outcome results were the same.

\section{Discussion}

Ninety-day hospital readmission rates are important patient safety indicators and potential drivers of quality improvement initiatives. Understanding of readmission incidences, demographics, and outcomes 
represents an opportunity to identify patients at risk for complications and opportunities for improved systems of care. In our study, one-fifth (21.7\%) of acute ischemic stroke (AIS) patients treated with IV-tPA were readmitted within 90 days of initial hospitalization, of which $20.6 \%$ were readmissions to non-index hospitals. Prior ischemic stroke studies have reported 7-12\% 30-day readmission rates [12-17]. A large ischemic stroke hospital registry documented readmission rates of $10 \%$ at 30 days, $17 \%$ at 90 days, $24 \%$ at 180 days, and $36 \%$ at 360 days [18]. IV-tPA treatment is clearly beneficial to properly selected acute stroke patients. However, its administration is associated with risk of post-treatment complications such as intracranial hemorrhage, angioedema, re-occlusion, secondary embolization, and neurotoxicity [19-22]. Recurrent strokes and seizures may also occur following TPA administration as a function of the initial stroke etiology or a complication related to the ischemic tissue bed [23, 24]. The most common cause for readmission within 30 days not related to IV-tPA is acute cerebrovascular disease, accounting for nearly $20 \%$ of readmitted patients [12, 17]. Previous nationwide studies in stroke suggest that infection is the second leading cause of 30 -day readmission (10-15\% of patients) $[12,14]$. Our results demonstrate a similar pattern, with cerebral artery occlusion (8.73\%), septicemia (5.83\%), and carotid artery occlusion (5.22\%) as the most common causes for readmission within 90 days. This suggests that while cerebrovascular disease may account for a large proportion of early readmissions, other underlying disease processes or complications may be important in disease progression.

Fragmentation of care and readmission to a non-index hospital has been associated with higher rates of morbidity and mortality in other disease processes [11, 25]. While our results indicate that readmission to a non-index hospital after IVT for AIS was associated with more frequent secondary readmissions, it did not result in worse outcomes. Overall, patients readmitted to index vs. non-index hospitals did not differ with regards to mortality, major complications, or neurological complications. This may be due to mature systems of stroke care and standardization of IV-tPA administration. Patients suspected of having a stroke are typically directed to a hospital that is capable of administering IV-tPA. These hospitals include acute stroke ready hospitals (ASRH) and primary stroke centers (PSC) that are uniquely equipped to diagnose and treat patients with an ischemic stroke. ASRH are responsible for stabilizing the patient, providing initial therapy, and arranging efficient transport to a higher level of care. PSCs are capable of managing the majority of acute stroke patients and have designated care teams, stroke units, protocols, and rapid imaging and laboratory services $[26,27]$. The methods and procedures for IV-tPA infusion and patient monitoring/ management are standardized and very well regulated according to guidelines. While there is likely variation to other procedures and ancillary services that an IV-tPA- treated stroke patient may have received, there is little ambiguity as to the thrombolysis treatment and related protocols at the time of initial stroke hospitalization. This makes it easier for medical teams to administer care and necessary treatments upon readmission. There may be less need for detailed history of the initial IV-tPA treatment, protocols, blood pressure parameters, and imaging paradigms. This is not true for many other disease processes where surgeries, chemotherapy regimens, or treatment protocols could vary greatly across disease types/ progression and different hospital systems. Standardization of acute stroke care across healthcare providers results in more comprehensive and coherent care of patients with ischemic stroke. The present study suggests that stroke systems of care are effective in the follow-up period, regardless of readmission destination.

This study has limitations. This study relies on retrospective data from the Nationwide Readmissions Database. Data is therefore subject to coding errors and information bias. The NRD includes only readmissions occurring in the same state as initial treatment and out-of-state readmissions are unable to be included in the analysis. There are a limited number of variables available in the NRD. Notably, race and ethnicity are not included, so the effect of these factors cannot be determined. Despite these limitations inherent to the database, the large sample size and use of multivariable analysis allows us to assess outcomes of 90-day readmissions.

\section{Conclusions}

Readmission to non-index hospital following IV-tPA treatment for acute stroke was not associated with increased mortality or major complications, unlike what has been seen in prior studies of other diseases processes. This difference may be due to standardized algorithms, mature systems of care, and demanding metrics required of acute stroke centers.

\section{Additional Information \\ Disclosures}

Human subjects: All authors have confirmed that this study did not involve human participants or tissue. Animal subjects: All authors have confirmed that this study did not involve animal subjects or tissue. Conflicts of interest: In compliance with the ICMJE uniform disclosure form, all authors declare the following: Payment/services info: NIH SC CTSI KL2 Clinical and Translational Research Scholar Award to FJA. Financial relationships: All authors have declared that they have no financial relationships at present or within the previous three years with any organizations that might have an interest in the submitted work. Other relationships: All authors have declared that there are no other relationships or activities that could appear to have influenced the submitted work. 


\section{References}

1. Adams HP, del Zoppo G, Alberts MJ, et al.: Guidelines for the early management of adults with ischemic stroke: a guideline from the American Heart Association/American Stroke Association Stroke Council, Clinical Cardiology Council, Cardiovascular Radiology and Intervention Council, and the Atherosclerotic Peripheral Vascular Disease and Quality of Care Outcomes in Research Interdisciplinary Working Groups: The American Academy of Neurology affirms the value of this guideline as an educational tool for neurologists. Circulation. 2007, 38:165-1711. https://doi.org/10.1161/STROKEAHA.107.181486

2. Shkirkova K, Saver JL, Starkman S, et al.: Frequency, predictors, and outcomes of prehospital and early postarrival neurological deterioration in acute stroke: exploratory analysis of the FAST-MAG Randomized clinical trial. JAMA neurology. 2018, 75:1364-74. doi:10.1001/jamaneurol.2018.1893

3. Siegler JE, Albright KC, George AJ, et al.: Time to neurological deterioration in ischemic stroke. Med Student Res J. 2017 Winter, 4:18-24. 10.15404/msri/03.2016.0005

4. National Institute of Neurological Disorders and Stroke rt-PA Stroke Study Group: Tissue plasminogen activator for acute ischemic stroke. N Engl J Med. 1995, 333:1581-7. DOI: 10.1056/NEJM199512143332401

5. Hacke W, Kaste M, Bluhmki E, et al.: Thrombolysis with alteplase 3 to 4.5 hours after acute ischemic stroke . N Engl J Med. 2008, 359:1317-29. 10.1056/NEJMoa0804656

6. Lees KR, Bluhmki E, von Kummer R, et al.: Time to treatment with intravenous alteplase and outcome in stroke: an updated pooled analysis of ECASS, ATLANTIS, NINDS, and EPITHET trials. Lancet. 2010, 375:1695-703. 10.1016/S0140-6736(10)60491-6

7. Jauch EC, Saver JL, Adams HP, et al.: Guidelines for the early management of patients with acute ischemic stroke: a guideline for healthcare professionals from the American Heart Association/American Stroke Association. Stroke. 2013, 44:870-947. 10.1161/STR.0b013e318284056a

8. Powers WJ, Rabinstein AA, Ackerson T, et al.: 2018 Guidelines for the early management of patients with acute ischemic stroke: a guideline for healthcare professionals from the American Heart Association/American Stroke Association. Stroke. 2018, 49:e46-110. https://doi.org/10.1161/STR.0000000000000158

9. Burke JF, Skolarus LE, Adelman EE, Reeves MJ, Brown DL: Influence of hospital-level practices on readmission after ischemic stroke. Neurology. 2014, 82:2196-204. 10.1212/WNL.0000000000000514

10. Burke RE, Jones CD, Hosokawa P, Glorioso TJ, Coleman EA, Ginde AA: Influence of nonindex hospital readmission on length of stay and mortality. Med care. 2018, 56:85-90. 10.1097/MLR.0000000000000829

11. Zafar SN, Shah AA, Channa H, Raoof M, Wilson L, Wasif N: Comparison of rates and outcomes of readmission to index vs nonindex hospitals after major cancer surgery. JAMA surgery. 2018, 153:719-27. 10.1001/jamasurg.2018.0380

12. Bambhroliya AB, Donnelly JP, Thomas EJ, et al.: Estimates and temporal trend for US nationwide 30-Day hospital readmission among patients with ischemic and hemorrhagic stroke. JAMA network open. 2018, 1:e181190. 10.1001/jamanetworkopen.2018.1190

13. Bhattacharya P, Khanal D, Madhavan R, Chaturvedi S: Why do ischemic stroke and transient ischemic attack patients get readmitted?. J Neurol Sci. 2011, 307:50-4. 10.1016/j.jns.2011.05.022

14. Boehme AK, Kulick ER, Canning M, et al.: Infections increase the risk of 30-Day readmissions among stroke survivors. Stroke. 2018, 49:2999-3005. 10.1161/STROKEAHA.118.022837

15. Mittal MK, Rabinstein AA, Mandrekar J, Brown RD, Jr., Flemming KD: A population-based study for 30-d hospital readmissions after acute ischemic stroke. Int J Neurosci. 2017, 127:305-13. 10.1080/00207454.2016.1207642

16. Suri MF, Qureshi AI: Readmission within 1 month of discharge among patients with acute ischemic stroke: results of the University HealthSystem Consortium Stroke Benchmarking study. J Vasc Interv Neurol. 2013, 6:47-51.

17. Vahidy FS, Donnelly JP, McCullough LD, et al.: Nationwide estimates of 30-day readmission in patients with ischemic stroke. Stroke. 2017, 48:1386-8. 10.1161/STROKEAHA.116.016085

18. Lin HJ, Chang WL, Tseng MC: Readmission after stroke in a hospital-based registry: risk, etiologies, and risk factors. Neurology. 2011, 76:438-43. 10.1212/WNL.0b013e31820a0cd8

19. Ali LK, Saver JL: The ischemic stroke patient who worsens: new assessment and management approaches . Rev Neurol Dis. 2007, 4:85-91.

20. Balami JS, Sutherland BA, Buchan AM: Complications associated with recombinant tissue plasminogen activator therapy for acute ischaemic stroke. CNS Neurol Disord Drug Targets. 2013, 12:155-69. 10.2174/18715273112119990050

21. Miller DJ, Simpson JR, Silver B: Safety of thrombolysis in acute ischemic stroke: a review of complications, risk factors, and newer technologies. Neurohospitalist. 2011, 1:138-47. 10.1177/1941875211408731

22. O'Carroll CB, Aguilar MI: Management of postthrombolysis hemorrhagic and orolingual angioedema complications. Neurohospitalist. 2015, 5:133-41. 10.1177/1941874415587680

23. Kumar S, Selim MH, Caplan LR: Medical complications after stroke. Lancet Neurol. 2010, 9:105-18. 10.1016/S1474-4422(09)70266-2

24. Rao A, Barrow E, Vuik S, Darzi A, Aylin P: Systematic review of hospital readmissions in stroke patients . Stroke Res Treat. 2016, 2016:9325368. 10.1155/2016/9325368

25. Zheng C, Habermann EB, Shara NM, et al.: Fragmentation of care after surgical discharge: non-Index readmission after major cancer surgery. J Am Coll Surg. 2016, 222:780-9. https://doi.org/10.1016/j.jamcollsurg.2016.01.052

26. Alberts MJ, Latchaw RE, Jagoda A, et al.: Revised and updated recommendations for the establishment of primary stroke centers: a summary statement from the brain attack coalition. Stroke. 2011, 42:2651-65. 10.1161/STROKEAHA.111.615336

27. Higashida R, Alberts MJ, Alexander DN, et al.: Interactions within stroke systems of care: a policy statement from the American Heart Association/American Stroke Association. Stroke. 2013, 44:2961-84. 10.1161/STR.0b013e3182a6d2b2 